\title{
The size of the solvable residual in finite groups
}

\author{
Silvio Dolfi, Marcel Herzog, Gil Kaplan and Arieh Lev
}

Dedicated to our colleague and friend Avinoam Mann

on the occasion of his retirement

\begin{abstract}
Let $G$ be a finite group. The solvable residual of $G$, denoted by $\operatorname{Res}(G)$, is the smallest normal subgroup of $G$ such that the respective quotient is solvable. We prove that every finite non-trivial group $G$ with a trivial Fitting subgroup satisfies the inequality $|\operatorname{Res}(G)|>$ $|G|^{\beta}$, where $\beta=\log (60) / \log \left(120(24)^{1 / 3}\right) \approx 0.700265861$. The constant $\beta$ in this inequality can not be replaced by a larger constant.
\end{abstract}

Mathematics Subject Classification (2000). 20B05, 20D25, $20 \mathrm{~F} 14$.

Keywords. Commutator subgroup, centre, Frattini subgroup, Fitting subgroup, solvable residual.

\section{Introduction}

All groups in this paper are finite. We use the standard notation $Z(G), \Phi(G), F(G)$ and $k(G)$ for the centre, the Frattini subgroup, the Fitting subgroup and the number of conjugacy classes of $G$, respectively. The solvable residual of $G, \operatorname{Res}(G)$, is the smallest normal subgroup of $G$ such that the respective quotient is solvable; this is the minimal term in the derived series of $G$. The nilpotent residual, $U(G)$, is defined similarly, and, of course, the commutator subgroup $G^{\prime}$ is the abelian residual of $G$. As usual, we denote by $S_{n}$ and $A_{n}$ the symmetric and alternating groups on $n$ letters.

In [5] and [7] the authors obtained the following bounds for the commutator subgroup and the nilpotent residual:

Theorem A. Let $G$ be a non-abelian group such that $\Phi(G)=1$. Then

$$
\left|G^{\prime}\right|>[G: Z(G)]^{1 / 2} .
$$


Theorem B. Let $G$ be a non-abelian group such that $\Phi(G)=1$ and let $U=U(G)$, the nilpotent residual of $G$. If $G$ is of odd order, then

$$
|U|>(2[G: Z(G)])^{1 / 2},
$$

and if $G$ is of even order not divisible by a Mersenne or a Fermat prime, then

$$
|U|>[G: Z(G)]^{1 / 2} .
$$

Other related results may be found in [1], [5], [7], [8] and [9]. In their recent paper [6], Guralnick and Robinson proved that $k(G) \leq(|F(G)||G|)^{1 / 2}$, which implies the inequality of Theorem A under the assumption that $F(G)=1$.

In this paper we prove a respective result for $\operatorname{Res}(G)$ :

Theorem C. Let $G \neq 1$ be a group such that $F(G)=1$. Then

$$
|\operatorname{Res}(G)|>|G|^{2 / 3} \text {. }
$$

More precisely,

$$
|\operatorname{Res}(G)|>|G|^{\beta}
$$

where $\beta=\log (60) / \log \left(120(24)^{1 / 3}\right) \approx 0.700265861$.

Theorem C improves the result of Proposition 2.4 in [7] $\left(|\operatorname{Res}(G)|>|G|^{1 / 2}\right.$ if $F(G)=1)$. In the proof of Theorem $\mathrm{C}$ we use the method of that proposition and the classification of the finite non-abelian simple groups. Both proofs rely heavily on J. D. Dixon's Theorem 3 in [3], where it is shown that the order of a solvable subgroup of $S_{n}$ is bounded by $24^{(n-1) / 3}$. The proof of this and related results can be also found in Section 5.8 of [4]; see also [11].

Remark 1. The assumption $F(G)=1$ can not be omitted in Theorem C. Furthermore, it is not enough to assume $\Phi(G)=1$ in Theorem C (unlike Theorem A and Theorem B). Indeed, by taking a direct product of a "large" elementary abelian group and a simple non-abelian group, one can find examples of non-solvable groups $G$ satisfying $\Phi(G)=1$ and $|\operatorname{Res}(G)|<|G|^{\varepsilon}$, for any $\varepsilon>0$.

Remark 2. The choice of the constant $\beta$ given in Theorem $\mathrm{C}$ is best possible, as the following example shows.

Example 1 (see [4], Exercise 5.8.6). Let $H=A_{5} \cong \operatorname{PSL}(2,4)$. Then $|H|=60$ and $|\operatorname{Aut}(H)|=120$. Denote $L_{4}=S_{4}, L_{16}=L_{4} \mathrm{wr} L_{4}$, and by induction: $L_{4^{k}}=L_{4^{k-1}}$ wr $L_{4}$ for every integer $k \geq 2$. Then $L_{4^{k}}$ is a solvable subgroup of $S_{4^{k}}$ of order $24^{\left(4^{k}-1\right) / 3}$. 
Denote $m=4^{k}$ and let $G_{m}=\operatorname{Aut}(H) \operatorname{wr} L_{m}$. Then $G_{m}$ is a subgroup of $\operatorname{Aut}\left(H^{m}\right)$ (see [12], Lemma 9.24), and one can verify that $F\left(G_{m}\right)=1$. Furthermore, we have $\operatorname{Res}\left(G_{m}\right)=\operatorname{Inn}(H)^{m}$, and so $\left|\operatorname{Res}\left(G_{m}\right)\right|=60^{m}$, while $\left|G_{m}\right|=$ $120^{m} 24^{(m-1) / 3}$.

Let $\beta_{m}=\log (60) / \log \left(120(24)^{\frac{m-1}{3 m}}\right)$. Then $\left|\operatorname{Res}\left(G_{m}\right)\right|=\left|G_{m}\right|^{\beta_{m}}$. Since $\left\{\beta_{m}\right\}$ is a decreasing sequence and since $\lim _{m \rightarrow \infty} \beta_{m}=\beta$, we have that for every $\varepsilon>0$ there exists an integer $m$ such that $\left|\operatorname{Res}\left(G_{m}\right)\right|<\left|G_{m}\right|^{\beta+\varepsilon}$. Thus the choice of $\beta$ in Theorem $\mathrm{C}$ is best possible.

\section{Proof of Theorem $\mathrm{C}$}

We will make use of the following proposition:

Proposition D. Let $S$ be a non-abelian simple group. Then, for every positive inte$\operatorname{ger} \alpha$,

$$
\left((24)^{\frac{\alpha-1}{3 \alpha}}|\operatorname{Out}(S)|\right)^{\frac{\beta}{1-\beta}}<|S|,
$$

where $\beta=\log (60) / \log \left(120(24)^{(1 / 3)}\right)$.

Proof. Since the sequence $\left\{\frac{\alpha-1}{3 \alpha}\right\}$ is an increasing sequence which converges to $1 / 3$, it suffices to show that

$$
\left((24)^{1 / 3}|\operatorname{Out}(S)|\right)^{\frac{\beta}{1-\beta}} \leq|S| .
$$

We note first that if $|\operatorname{Out}(S)|=2,3$ or 4 , then, for the above inequality to hold, it suffices that the value of $|S|$ will be at least 60,155 and 304, respectively. Note further that $24^{1 / 3}<2.9$ and $\frac{\beta}{1-\beta}<2.4$. We will use the above values in the sequel.

In the following, we refer to tables 5.1.A, 5.1.B and 5.1.C in [10].

First, observe that if $S$ is sporadic or alternating, then $|\operatorname{Out}(S)| \leq 2$, with the exception $\left|\operatorname{Out}\left(A_{6}\right)\right|=4$ (see [2], remark on p. ix and Table 1). Then, since $|S| \geq 60$ and $\left|A_{6}\right|=360$, we are done in this case.

Hence, we are left with the simple groups of Lie type over a field of $q$ elements. As in [10], we denote $q=p^{f}$, where $p$ is a prime. We start with the simple classical groups and consider the following cases:

I) $S=A_{n-1}(q)$ (i.e. $\left.S=\operatorname{PSL}_{n}(q)\right), n \geq 2$. Here

$$
|S|=\frac{1}{d} q^{\frac{n(n-1)}{2}} \prod_{i=2}^{n}\left(q^{i}-1\right) \quad \text { and } \quad|\operatorname{Out}(S)|= \begin{cases}d f & \text { if } n=2 \\ 2 d f & \text { if } n \geq 3\end{cases}
$$

where $d=(n, q-1)$.

We consider first the case $n \geq 3$. Using the fact that $d \leq q-1$ and by the remark at the beginning of the proof, it suffices to show that $(2.9 \cdot 2(q-1) f)^{2.4} \leq|S|$. 
Since the left hand side of the inequality is independent of $n$ and since the right hand side is an increasing function of $n$, it suffices to show that $(2.9 \cdot 2(q-1) f)^{2.4} \leq$ $\frac{1}{q-1} q^{3}\left(q^{2}-1\right)\left(q^{3}-1\right)=\frac{1}{q-1}(q-1)^{2} q^{3}(q+1)\left(q^{2}+q+1\right)$, and in particular, it is enough to show that $(2.9 \cdot 2(q-1) f)^{2.4} \leq(q-1) q^{6}$. Hence, it suffices to show that $(5.8 f)^{2.4} \leq q^{4.6}=p^{4.6 f}$. This inequality holds for all integers $p \geq 2$ and $f \geq 1$ with the only exception $p=2$ and $f=1$ (i.e. $q=2$ ). However, in that case we have $|\operatorname{Out}(S)|=2,|S|=168$ and the result follows.

Consider now the case $n=2$. Since $S$ is not simple for $q=2,3$, we consider the case $q \geq 4$. Since $|\operatorname{Out}(S)|=2$ for $q=4,5,7$ (while of course $|S| \geq 60$ ), and since $|\operatorname{Out}(S)| \leq 4$ for $q \leq 25$ (while $|S| \geq 304$ for $q \geq 8$ ), it is enough to check the case $q \geq 27$. As before, it suffices to show that $(2.9 \cdot d f)^{2.4} \leq$ $|S|=\frac{1}{d} q\left(q^{2}-1\right)=\frac{1}{d} p^{f}\left(p^{2 f}-1\right)$. If $p=2$ then $d=1$ and (by the above considerations) $f \geq 5$, while the last inequality reduces to $(2.9 f)^{2.4} \leq 2^{f}\left(2^{2 f}-1\right)$ which holds for every $f \geq 5$. Therefore, we may assume that $p \geq 3$. Furthermore, if $p=3$ or 5 then $f \geq 3$, while $f \geq 1$ otherwise. Since the inequality $(5.8 f)^{2.4} \leq$ $\frac{1}{2} p^{f}\left(p^{2 f}-1\right)$ holds when either $\bar{p}=3$ or 5 and $f \geq 3$ or $p \geq 7$ and $f \geq 1$, the result follows.

II) $S={ }^{2} A_{n-1}(q)=U_{n}(q)(n \geq 2)$. Here we have

$$
|S|=\frac{1}{d} q^{\frac{n(n-1)}{2}} \prod_{i=2}^{n}\left(q^{i}-(-1)^{i}\right) \quad \text { and } \quad|\operatorname{Out}(S)|= \begin{cases}d f & \text { if } n=2 \\ 2 d f & \text { if } n \geq 3\end{cases}
$$

where $d=(n, q+1)$.

If $n=2$, then $|S|$ and $|\operatorname{Out}(S)|$ are the same as in the previous case. Assume now that $n=3$. Then $|S|=\frac{1}{d} q^{3}\left(q^{2}-1\right)\left(q^{3}+1\right)$, where $d \in\{1,3\}$. If $q=2$ then $|S|=72$ and $S$ is not simple. When $q \geq 3$ it is enough to set $d=3$ (the "worst case") and check the inequality $(2.9 \cdot 2 \cdot 3 f)^{2.4} \leq \frac{1}{3} q^{7}=\frac{1}{3} p^{7 f}$, which is easily verified for every $p \geq 3, f \geq 2$. When $p=3$ and $f=1$ we have $|\operatorname{Out}(S)|=2$, and so we may apply the remark at the beginning of the proof.

If $n \geq 4$, then it is enough to check (the "worst case" $d=q+1$ ) that $(2.9 \cdot 2$. $(q+1) f)^{2.4} \leq \frac{1}{q+1} q^{6}\left(q^{2}-1\right)\left(q^{3}+1\right)\left(q^{4}-1\right)$. Thus it certainly suffices to check that $(5.8 \cdot 2 q f)^{2.4} \leq q^{12}$, i.e. $\left(5.8 \cdot 2 p^{f} f\right)^{2.4} \leq p^{12 f}$ for every $p \geq 2, f \geq 1$. This inequality is easily verified and so we are done in this case.

III) Either $S=\operatorname{PSp}_{2 m}(q)=C_{m}(q)$ (where $m \geq 2$ ) or $S=\Omega_{2 m+1}(q)=B_{m}(q)$ (where $m \geq 2$ and $q$ is odd). Here we have

$$
|S|=\frac{1}{d} q^{m^{2}} \prod_{i=1}^{m}\left(q^{2 i}-1\right) \quad \text { and } \quad|\operatorname{Out}(S)|= \begin{cases}2 f & \text { if } m=2 \\ d f & \text { if } m \geq 3\end{cases}
$$

with $d=(2, q-1)$ if $S=\operatorname{PSp}_{2 m}(q)$, while $d=2$ if $S=\Omega_{2 m+1}(q)$. Except the case $S=\operatorname{PSp}_{4}(q)$ (where $m=2$ ) with $q$ even, it is clear that $|S|$ exceeds $\left|\operatorname{PSL}_{m}(q)\right|$, 
while $|\operatorname{Out}(S)|$ does not exceed $\left|\operatorname{Out}\left(\operatorname{PSL}_{m}(q)\right)\right|$. Thus, with the above exception, the validity of the required inequality follows by the arguments given in case I. However, in the case $S=\operatorname{PSp}_{4}(q)$ we have $|\operatorname{Out}(S)|=2 f$ and $|S|=p^{4 f}\left(p^{2 f}-1\right)\left(p^{4 f}-1\right)$. Since the inequality $(2.9 \cdot 2 f)^{2.4} \leq p^{4 f}\left(p^{2 f}-1\right)\left(p^{4 f}-1\right)$ holds for every $p \geq 2$ and $f \geq 1$ (it suffices to check the case $p=2$ and $f=1$ ), the checking of this case is completed.

IV) Either $S=P \Omega_{2 m}^{+}(q)=D_{m}(q)$, where $m \geq 3$ and

$$
|S|=\frac{1}{d} q^{m(m-1)}\left(q^{m}-1\right) \prod_{i=1}^{m-1}\left(q^{2 i}-1\right) \quad \text { and } \quad|\operatorname{Out}(S)|= \begin{cases}2 d f & \text { if } m \neq 4 \\ 6 d f & \text { if } m=4\end{cases}
$$

with $d=\left(4, q^{m}-1\right)$, or $S=P \Omega_{2 m}^{-}(q)={ }^{2} D_{m}(q)$, where $m \geq 2$ and

$$
|S|=\frac{1}{d} q^{m(m-1)}\left(q^{m}+1\right) \prod_{i=1}^{m-1}\left(q^{2 i}-1\right) \quad \text { and } \quad|\operatorname{Out}(S)|=2 d f
$$

with $d=\left(4, q^{m}+1\right)$.

We notice that in this case some of the groups are isomorphic to groups discussed in previous cases (see [2], p. xii). However, for the convenience of the reader we provide a direct argument for all the groups considered.

Consider first the case that $q=2$. Then, except the case $S=P \Omega_{2 m}^{+}(q), m=4$, we have $|\operatorname{Out}(S)|=2$, and so we are done by the remark at the beginning of the proof. If $S=P \Omega_{2 m}^{+}(q), m=4$, then $|\operatorname{Out}(S)|=6$, while $|S|=2^{12}\left(2^{4}-1\right)$. $\left(2^{2}-1\right)\left(2^{4}-1\right)\left(2^{6}-1\right)$. Since $(2.9 \cdot 6)^{2.4} \leq|S|$, the case that $q=2$ is completed.

The case $S=P \Omega_{2 m}^{+}(q), m=4$ for $q \geq 3$ is checked by verifying the inequality $(2.9 \cdot 24 f)^{2.4} \leq \frac{1}{4} p^{12 f}\left(p^{4 f}-1\right)\left(p^{2 f}-1\right)\left(p^{4 f}-1\right)\left(p^{6 f}-1\right)$ for every $p \geq 3, f \geq 1$ and for $p=2, f \geq 2$.

Assume now $q=3, S=P \Omega_{2 m}^{-}(q), m=2$. Then $|\operatorname{Out}(S)|=4$ and $|S|=$ $360 \geq 304$, hence the result follows by the remark at the beginning of the proof. The case $q=3, m \geq 3$ for $P \Omega_{2 m}^{+}(q)$ and $P \Omega_{2 m}^{-}(q)$ (excluding the possibility $S=P \Omega_{2 m}^{+}(q), m=4$, which was already done) is done by verifying the inequality $(2.9 \cdot 8)^{2.4} \leq \frac{1}{4} 3^{6} \cdot\left(3^{3}-1\right) \cdot\left(3^{2}-1\right) \cdot\left(3^{4}-1\right)$ (the right hand side is a lower bound for $|S|$ in this case).

In the case $q=4$, for $P \Omega_{2 m}^{+}(q)$ and $P \Omega_{2 m}^{-}(q)$ (excluding the possibility $S=$ $P \Omega_{2 m}^{+}(q), m=4$, which was already done), we have $d=1,|\operatorname{Out}(S)|=4$, and so it suffices to verify the inequality $(2.9 \cdot 4)^{2.4} \leq 4^{2} \cdot\left(4^{2}+1\right) \cdot\left(4^{2}-1\right)$ (the right hand side is a lower bound for $|S|$ ).

It is left to consider the case $q \geq 5$. Since the inequality $(2.9 \cdot 8 f)^{2.4} \leq$ $\frac{1}{4} p^{f m(m-1)}\left(p^{f m}-1\right) \prod_{i=1}^{m-1}\left(p^{2 f i}-1\right)$ holds for every $p \geq 5, m \geq 2, f \geq 1$ (it suffices to check for $p=5, m=2, f=1$ ), we are done. Hence case IV is completed. 
It remains to consider the simple exceptional groups. Assume first that $|\operatorname{Out}(S)|=$ $f$ (see [10], Table 5.1.B). Then $|S| \geq q^{4}=p^{4 f}$. Hence, we have only to consider the inequality $(2.9 f)^{2.4} \leq p^{4 f}$. Since this inequality holds for every $p \geq 2$ and $f \geq 1$, we are done in this case. Assume now that $|\operatorname{Out}(S)|>f$. Then we observe (Table 5.1.B again) that $|\operatorname{Out}(S)| \leq 6 f$ and $|S| \geq q^{12}$ in this case. Hence, since the inequality $(1.9 \cdot 6 f)^{2.4} \leq q^{12}=p^{12 f}$ holds for every $p \geq 2$ and $f \geq 1$, the proof of Proposition D is completed.

Proof of Theorem $C$. Apply induction on $|G|$. Let $N$ be a minimal normal subgroup of $G$ and let $N_{1} / N$ be the maximal normal solvable subgroup of $G / N$. Then $F\left(G / N_{1}\right)=1$. Suppose first that $N_{1}<G$. Then from $F\left(N_{1}\right) \leq F(G)=1$ it follows by induction that

$$
\left|\operatorname{Res}(G) \cap N_{1}\right| \geq\left|\operatorname{Res}\left(N_{1}\right)\right|>\left|N_{1}\right|^{\beta} .
$$

By applying further the inductive hypothesis to $G / N_{1}$, we obtain

$$
\left[\operatorname{Res}(G): \operatorname{Res}(G) \cap N_{1}\right]=\left|\operatorname{Res}(G) N_{1} / N_{1}\right|=\left|\operatorname{Res}\left(G / N_{1}\right)\right|>\left|G / N_{1}\right|^{\beta} .
$$

Now from (1) and (2) follows $|\operatorname{Res}(G)|>|G|^{\beta}$, as claimed.

Thus we may assume from now on that $N_{1}=G$, i.e., $G / N$ is solvable and $\operatorname{Res}(G)=N=T^{\alpha}$, a direct product, where $T$ is a simple non-abelian group (recall that $F(G)=1)$ and $\alpha \geq 1$ is an integer. We notice that $N$ is the unique minimal normal subgroup of $G$. Indeed, suppose on the contrary that there exists another minimal normal subgroup, say $M$, of $G$. Then $M \cap N=1$ and $M$ is embedded in the solvable group $G / N$, contradicting $F(G)=1$.

We deduce that $C_{G}(N)=1$ and $N \leq G \leq \operatorname{Aut}(N)=\operatorname{Aut}(T)$ wr $S_{\alpha}$ (see [12], Lemma 9.24). Thus $G / N$ is a solvable group embedded in $\operatorname{Out}(T) \mathrm{wr} S_{\alpha}$. Any element of $G / N$ has the form $(b, \sigma)$, where $b$ belongs to the base subgroup of $\operatorname{Out}(T) \operatorname{wr} S_{\alpha}$ and $\sigma \in S_{\alpha}$. Then the function $(b, \sigma) \mapsto \sigma$ is a homomorphism from $G / N$ into $S_{\alpha}$. Denote the image of this homomorphism by $D$. Then $D$ is a solvable subgroup of $S_{\alpha}$ and thus, by [3], Theorem 3, $|D| \leq f(\alpha):=24^{(\alpha-1) / 3}$. Since $|G / N| \leq|\operatorname{Out}(T)|^{\alpha}$. $|D|$, it follows that $|G / N| \leq|\operatorname{Out}(T)|^{\alpha} \cdot f(\alpha)$. Since $\operatorname{Res}(G)=N$ and since we want to show that $|N|>|G|^{\beta}$, it suffices to show that $|G / N|<|N|^{\frac{1-\beta}{\beta}}=|T|^{\alpha \frac{1-\beta}{\beta}}$. For that, it is enough to check that $|\operatorname{Out}(T)| f(\alpha)^{1 / \alpha}<|T|^{\frac{1-\beta}{\beta}}$ for each simple nonabelian group $T$. Thus, it suffices to show that $\left(24^{\frac{\alpha-1}{3 \alpha}}|\operatorname{Out}(T)|\right)^{\frac{\beta}{1-\beta}}<|T|$ holds for every positive integer $\alpha$ and every non-abelian simple group $T$. Since this inequality holds by Proposition D, the proof of the theorem is complete. 


\section{References}

[1] M. Bianchi, A. Gillio, H. Heineken, and L. Verardi, Groups with big centralizers. Istit. Lombardo Accad. Sci. Lett. Rend. A 130 (1996), 25-42 (1997). MR 1620400

[2] J. H. Conway, R. T. Curtis, S. P. Norton, R. A. Parker, and R. A. Wilson, Atlas of finite groups. Oxford University Press, Oxford 1985. Zbl 0568.20001 MR 0827219

[3] J. D. Dixon, The Fitting subgroup of a linear solvable group. J. Austral. Math. Soc. 7 (1967), 417-424. Zbl 0153.04001 MR 0230814

[4] J. D. Dixon and B. Mortimer, Permutation groups. Grad. Texts in Math. 163, SpringerVerlag, New York 1996. Zbl 0951.20001 MR 1409812

[5] S. Dolfi, M. Herzog, G. Kaplan, and A. Lev, On the size of the nilpotent residual in finite groups. J. Group Theory 10 (2007), 299-305. Zbl 05166574 MR 2320969

[6] R. M. Guralnick and G. R. Robinson, On the commuting probability in finite groups. J. Algebra 300 (2006), 509-528. Zbl 1100.20045 MR 2228209

[7] M. Herzog, G. Kaplan, and A. Lev, The size of the commutator subgroup of a finite group, J. Algebra, to appear.

[8] G. Kaplan and A. Lev, The existence of large commutator subgroups in factors and subgroups of non-nilpotent groups. Arch. Math. (Basel) 85 (2005), 197-202. Zbl 1093.20009 MR 2172377

[9] G. Kaplan and A. Lev, On groups satisfying $\left|G^{\prime}\right|>[G: Z(G)]^{1 / 2}$. Beiträge Algebra Geom. 47 (2006), 271-274. Zbl 1102.20021 MR 2246538

[10] P. Kleidman and M. Liebeck, The subgroup structure of the finite classical groups. London Math. Soc. Lecture Note Ser. 129, Cambridge University Press, Cambridge 1990. Zbl 0697.20004 MR 1057341

[11] P. P. Pálfy, A polynomial bound for the orders of primitive solvable groups. J. Algebra 77 (1982), 127-137. Zbl 0489.20004 MR 665168

[12] J. S. Rose, A course on group theory. Cambridge University Press, Cambridge 1978; reprinted by Dover Publications, New York, 1994. Zbl 0371.20001 MR 0498810

Received December 13, 2006; revised January 25, 2007

S. Dolfi, Dipartimento di Matematica “U. Dini”, Università degli Studi di Firenze, Firenze, 50134 Italy

E-mail: silvio.dolfi@math.unifi.it

M. Herzog, School of Mathematical Sciences, Raymond and Beverly Sackler Faculty of Exact Sciences, Tel-Aviv University, Tel-Aviv 69978, Israel

E-mail: herzogm@post.tau.ac.il

G. Kaplan, School of Computer Sciences, The Academic College of Tel-Aviv-Yaffo, 4 Antokolsky St., Tel-Aviv 64044, Israel

E-mail: gilk@mta.ac.il

A. Lev, School of Computer Sciences, The Academic College of Tel-Aviv-Yaffo, 4 Antokolsky St., Tel-Aviv 64044, Israel

E-mail: arieh@mta.ac.il 